\title{
Dolor neuropático localizado
}

\author{
Ricardo Plancarte-Sánchez, * Marcela Samano-García, María del Rocío Guillén-Núñez y \\ Antonio Equihua-Ortega \\ Instituto Nacional de Cancerología, Clínica del Dolor, Ciudad de México, México
}

\section{Resumen}

El dolor neuropático localizado (DNL) es de origen periférico y se caracteriza por áreas circunscritas de dolor con sensibilidad anormal de la piel o síntomas espontáneos característicos de dolor neuropático, por ejemplo, dolor urente. Se debe resaltar que el DNL está confinado a un área específica no mayor a una hoja de papel tamaño carta. El DNL representa $60 \%$ de las condiciones de dolor neuropático. No existe una única etiología. El abordaje diagnóstico es similar al de otros síndromes dolorosos neuropáticos. Se utilizan herramientas diagnósticas generales para evaluar las características clínicas. En la actualidad no existen guías específicas de manejo del DNL, por lo que se utilizan las guías para dolor neuropático en general. En las guías de la Sociedad Canadiense de Dolor se incluyen los tratamientos tópicos como parte de las estrategias de segunda línea. Pese a la falta de guías, los parches de lidocaína a 5 \% y los parches de capsaicina a 8 \% han demostrado ser efectivos en modelos de DNL.

PALABRAS CLAVE: Dolor neuropático localizado. Tratamiento tópico del dolor. Parche de lidocaína. Parches de capsaicina.

\section{Localized neuropathic pain}

\begin{abstract}
Localized neuropathic pain (LNP) is of peripheral origin and is characterized by circumscribed areas of pain with abnormal skin sensitivity or spontaneous symptoms that are characteristic of neuropathic pain, e.g. burning pain. It should be noted that LNP is confined to a specific area no larger than a letter size sheet of paper. LNP accounts for $60 \%$ of neuropathic pain conditions. There is no single etiology of LNP. The diagnostic approach is similar to that for other neuropathic pain syndromes. General diagnostic tools are used to assess clinical features. So far, there are no specific guidelines for the management of LNP; for this reason, guidelines for general neuropathic pain are used. Topical treatments are included as part of second-line strategies in the Canadian Pain Society guidelines. Despite the lack of guidelines, $5 \%$ lidocaine patches and $8 \%$ capsaicin patches have been proven effective in LNP models.
\end{abstract}

KEY WORDS: Localized neuropathic pain. Pain topical treatment. Lidocaine patch. Capsaicin patches.

\section{Introducción}

El dolor neuropático es definido como el dolor secundario a una enfermedad o lesión que afecta al sistema nervioso somatosensorial. ${ }^{1-3}$ Este tipo de dolor tiene varias formas de presentación: puede ser continuo o episódico recurrente y el cuadro clínico puede hacer referencia a modelos de dolor evocado o espontáneo. Entre los descriptores de dolor neuropático se encuentran ardor, hormigueo, hiperalgesia, alodinia, sensación de descargas eléctricas, dolor al
Correspondencia:

*Ricardo Plancarte-Sánchez

E-mail: planky2b@yahoo.com.mx
Fecha de recepción: 09-11-2020

Fecha de aceptación: 17-11-2020

DOI: 10.24875/GMM.20000810
Gac Med Mex. 2021;157:315-322

Disponible en PubMed

www.gacetamedicademexico.com

0016-3813/C 2020 Academia Nacional de Medicina de México, A.C. Publicado por Permanyer. Este es un artículo open access bajo la licencia CC BY-NC-ND (http://creativecommons.org/licenses/by-nc-nd/4.0/). 
frío o calor y entumecimiento. ${ }^{1}$ Tanto la expresión de mayor sensación a un estímulo que puede condicionar dolor (hiperalgesia) o la respuesta de dolor a un estímulo inocuo (alodinia) son descriptores objetivos que pueden acompañar al dolor neuropático. ${ }^{4}$

En cuanto a la temporalidad, el dolor neuropático puede ser agudo o crónico; la duración del primero es menor a tres meses y la del segundo, mayor a ese tiempo.

De acuerdo con el nivel de afección del sistema nervioso, el dolor neuropático se divide en dolor neuropático central, cuando hay afección medular 0 supramedular, y periférico, cuando la afección es previa al relevo medular. ${ }^{5,6}$

El dolor neuropático localizado (DNL) es de origen periférico y se caracteriza por áreas circunscritas de dolor con sensibilidad anormal de la piel o síntomas espontáneos característicos de dolor neuropático, por ejemplo, dolor urente. Además, se debe resaltar que el DNL está confinado a un área específica no mayor a una pieza de papel tamaño carta. ${ }^{6,7}$

La prevalencia del dolor neuropático periférico es de aproximadamente $2 \%$ en la población en general y de $8 \%$ en pacientes de más de 55 años de edad. ${ }^{8}$ La neuropatía diabética es la principal causa del dolor neuropático en el mundo. ${ }^{9,10}$ El DNL representa $60 \%$ de las condiciones de dolor neuropático. ${ }^{6}$

Las principales causas de DNL se asocian a las siguientes condiciones: : $^{10,11}$

- Infecciones (virus varicela zóster o virus de la inmunodeficiencia humana).

- Enfermedades metabólicas (diabetes mellitus, alcoholismo, déficit de vitamina $\mathrm{B}_{12}$ ).

- Déficits carenciales.

- Causas tóxicas (etanol, quimioterapia).

- Cirugías (toracotomía o mastectomía, cesárea, artroscopia o artroplastia, safenectomía, cirugía inguinal, colecistectomía, entre otras).

- Atrapamientos nerviosos (neuroma de Morton, síndrome del túnel del tarso, síndrome del túnel del carpo).

- Afección de la raíz nerviosa (secuelas de radiculalgia, dolor lumbar, neuralgia, síndrome cervicobraquial).

- Síndrome doloroso regional complejo tipo 1.

La características cualitativas del dolor neuropático ocasionan la suposición de este trastorno; sin embargo, el reciente desarrollo de cuestionarios diagnósticos han ayudado a la detección de componentes clínicos de origen neuropático de manera simple, rápida y específica. Estas herramientas de cribado son el cuestionario DN4, painDETECT, Neuropathic Pain Symptom
Tabla 1. Manejo farmacológico paso a paso del dolor neuropático

1 Evaluación y establecimiento del diagnóstico

- Establecer y tratar la causa de dolor neuropático

- Identificar comorbilidades relevantes

- Explicar el diagnóstico, plan y tratamiento al paciente

2 Inicio de tratamiento sintomático con una o más de las siguientes opciones:

- Antidepresivos selectivos de la recaptura de serotonina y noradrenalina

- Ligandos de los canales de calcio $\alpha 2-\delta$-dependiente

- Parches de lidocaína a $5 \%$

- Analgésicos opioides o tramadol, en circunstancias clínicas selectas

- Tratamientos no farmacológicos, previa evaluación

3 Reevaluación del dolor y la calidad de vida relacionada con la salud

- Si el dolor sustancialmente se alivió y los efectos adversos secundarios son tolerables, continuar el tratamiento

- Si el dolor mejoró parcialmente, ajustar la dosis inicial del medicamento 0 adicionar otro de primera línea

- Si no hay adecuado control del dolor, cambiar a otro medicamento de primera línea

4 Si fallan los tratamientos farmacológicos de primera y segunda línea, considerar medicamentos de primera línea o referir a un especialista en dolor.

Fuente: Dworkin RH, O'Connor AB, Backonja M, Farrar JT, Finnerup NB, Jensen TS, et al. Pharmacologic management of neuropathic pain: evidence-based recommendations. ${ }^{16}$

Inventory (NPSI) y la escala LANSS. ${ }^{5}$ Para el diagnóstico clínico del DNL también existe una herramienta denominada Diagnostic Tool. ${ }^{4,6,13,14}$ En general, el diagnóstico del dolor neuropático es a través de la historia clínica, el examen físico y las pruebas confirmatorias. ${ }^{14}$

Por su parte, se han propuesto tres etapas para el manejo del DNL: 2,5

1. Cribado y diagnóstico

2. Tratamiento

3. Modelo de seguimiento

Una vez realizado el diagnóstico clínico, el tratamiento del dolor neuropático está encaminado a la disminución de la sintomatología y mejoría en la funcionalidad del paciente (Figura 1). ${ }^{7,10,15}$

Existen diferentes publicaciones en las cuales se hace referencia al algoritmo farmacológico del paciente con dolor neuropático (Tabla 1). ${ }^{16,17}$

Es importante puntualizar que si el paciente presenta hiperalgesia extrema o sintomatología incontrolable pese a un tratamiento idóneo, se recomienda realizar la referencia a un especialista, sin importar la etapa del tratamiento y de atención, para una reevaluación diagnóstica. $^{2}$ 


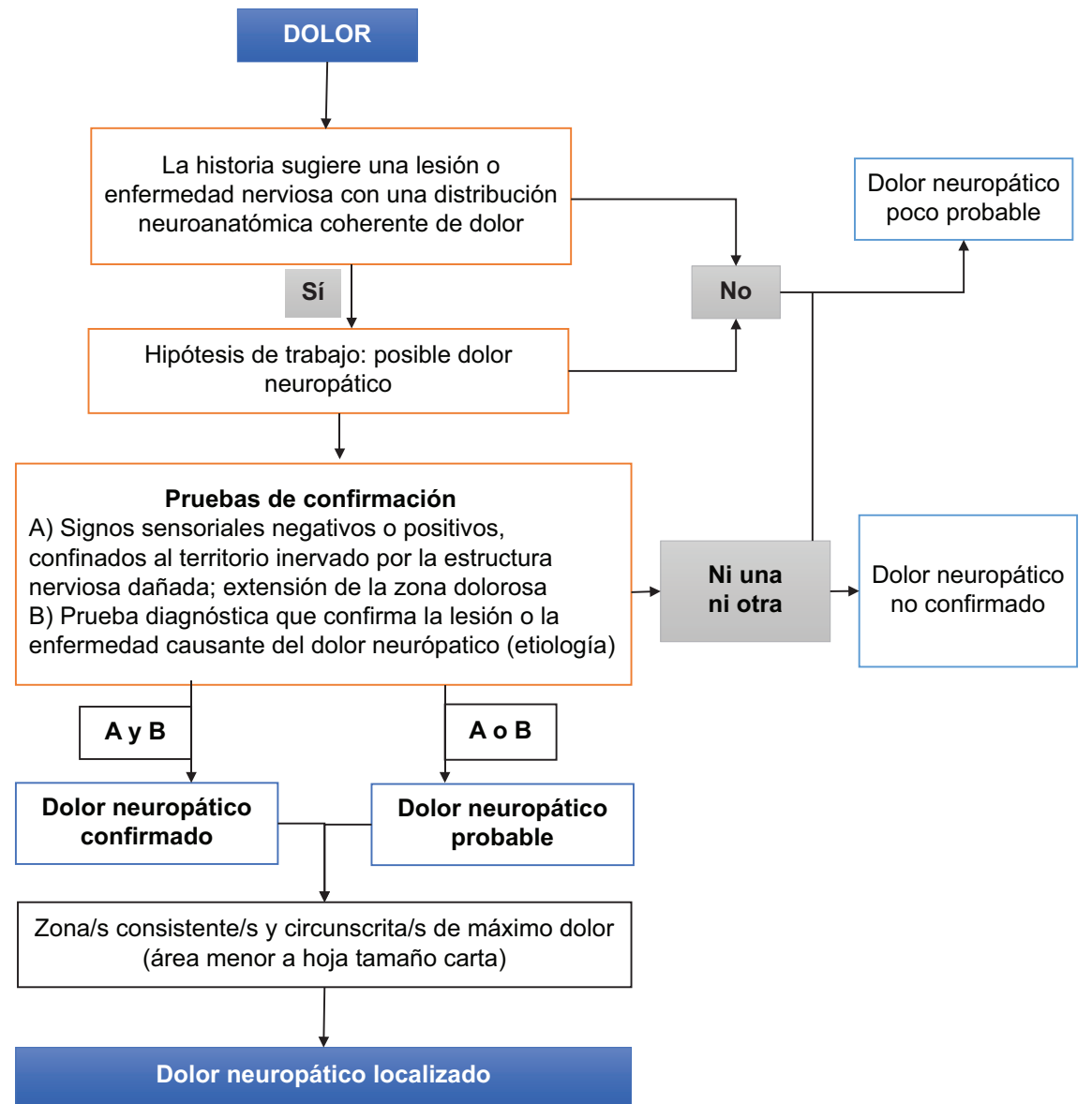

Figura 1. Herramienta de detección de dolor neuropático localizado. Modificado de Mick G et al.: Is an easy and reliable diagnosis of localized neuropathic pain (LNP) possible in general practice? Development of a screening tool based on IASP criteria. ${ }^{10}$

No existen guías específicas ni recomendaciones de tratamiento para el DNL, por lo que se utilizan las que existen para dolor neuropático en general. $^{12}$

La Federación Europea de la Sociedad Neurológica y la Asociación Internacional para el Estudio del Dolor recomienda el uso de antidepresivos tricíclicos, anticonvulsivos e inhibidores de la recaptura de serotonina y noradrenalina como primera opción en el tratamiento de la neuropatía periférica; sin embargo, la efectividad se presenta en aproximadamente 30 a $40 \%$ de los pacientes, lo cual tiene relación con los efectos adversos de esos fármacos, que disminuyen la calidad de vida. ${ }^{5,18,19,20}$

Las guías de la Sociedad Canadiense del Dolor refieren que para el DNL, además de los tratamientos convencionales, en segunda línea de tratamiento se incluye el uso tópico de lidocaína. ${ }^{17}$

Cabe mencionar que los antiinflamatorios no esteroideos y el paracetamol no han mostrado utilidad en el tratamiento del dolor neuropático. ${ }^{5}$
El manejo del dolor neuropático es un reto clínico y terapéutico, que en ocasiones no muestra resultados favorables durante meses 0 , incluso, años. Por esta razón, diferentes sociedades, incluidos el Grupo de Interés Especial en Dolor Neuropático (Neupsig, de la Asociación Internacional para el Estudio del Dolor), la Sociedad Europea del Dolor y la Sociedad Canadiense del Dolor, han propuesto tratamientos escalonados para el manejo del dolor neuropático crónico y niveles de evidencias, que se describen en la Tabla 2. 2,5,12,19,20,21

En el caso particular del tratamiento del DLN, está justificado el uso de agentes tópicos, a menos de que exista alguna contraindicación como lesión, atrofia o infección del área del cuerpo afectada. ${ }^{16,30,31}$ Este modelo terapéutico ofrece los siguientes beneficios: $:$,15,31,32

- Disminuye el DLN y evita los efectos adversos que podrían presentarse con el tratamiento oral. ${ }^{16}$

- Las dosis son menores a las dosis totales de los fármacos por vía oral. ${ }^{2}$ 

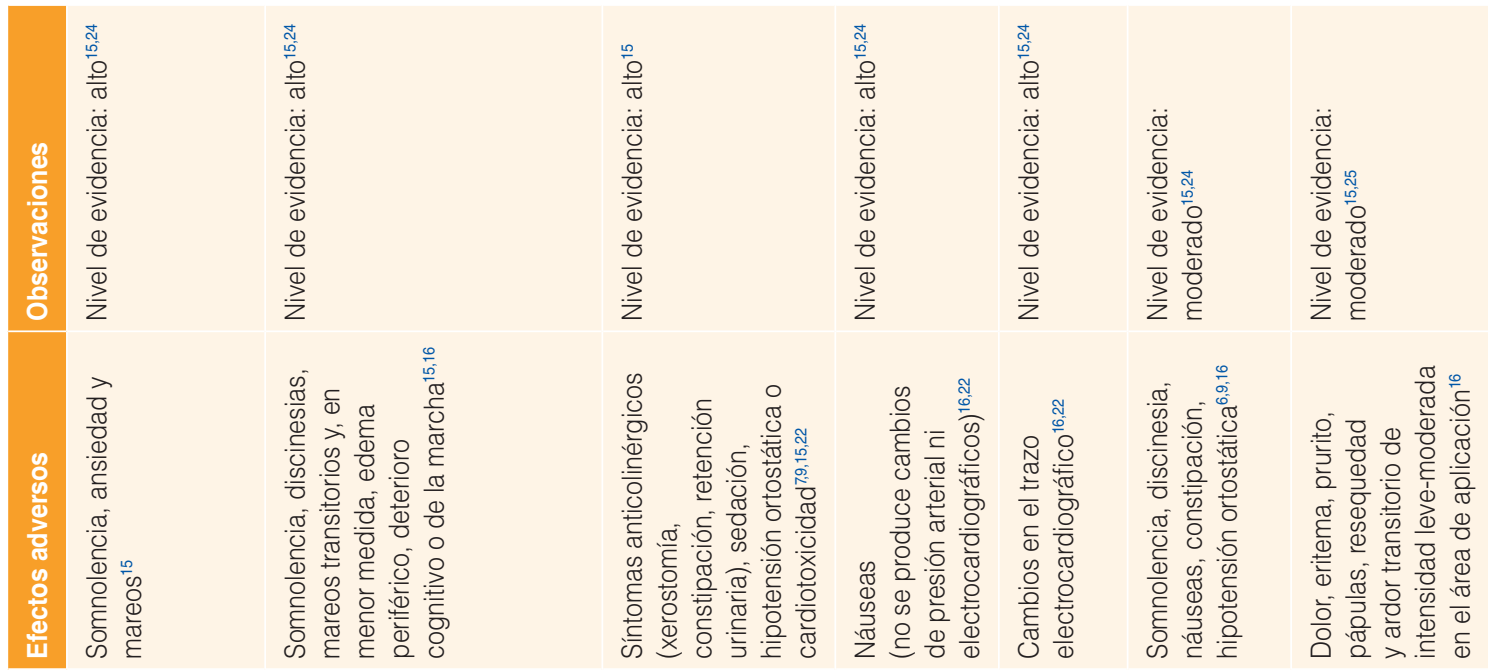

흥 뚜유

주응 은 응

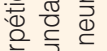

क्ष

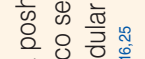
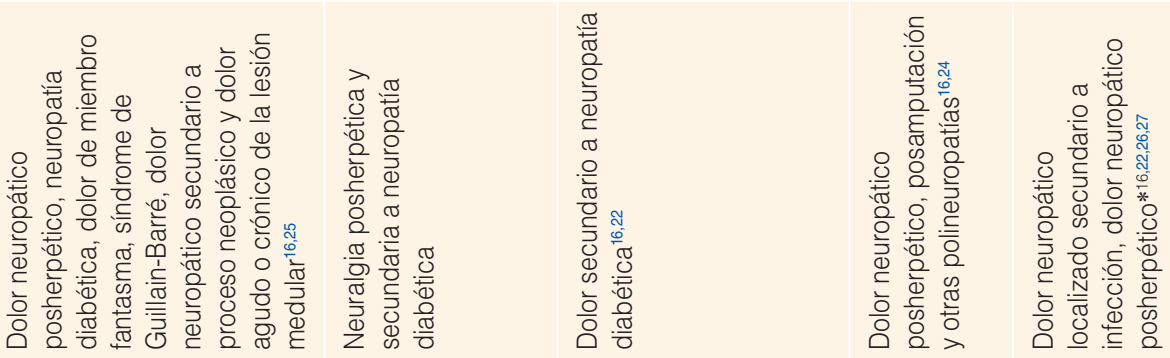

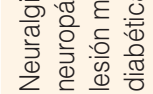
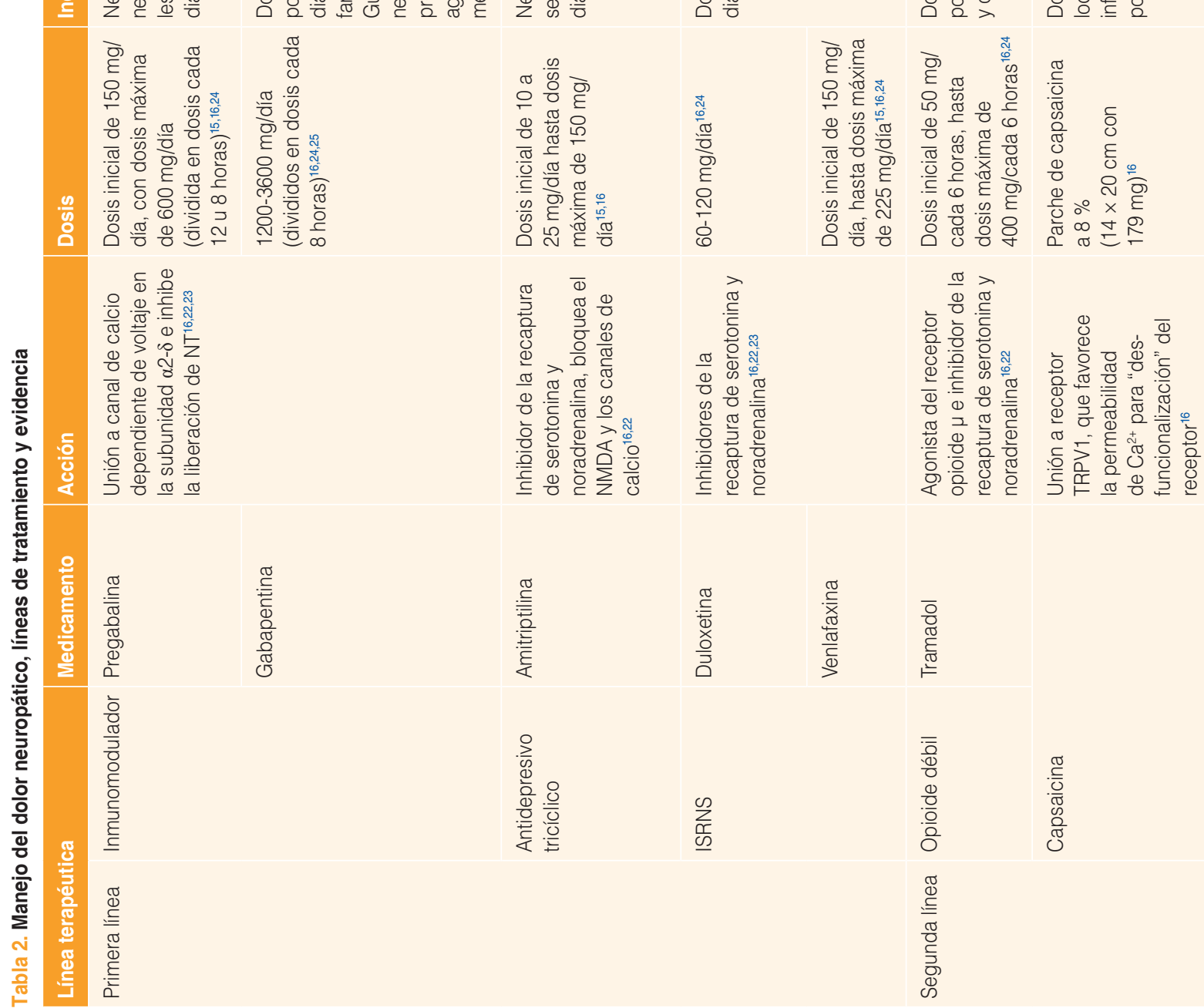

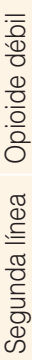
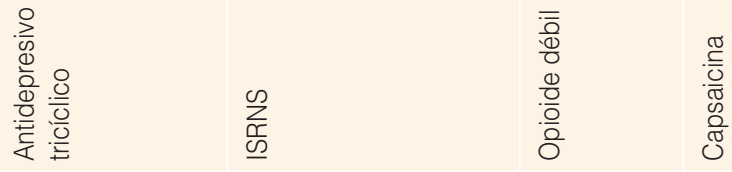


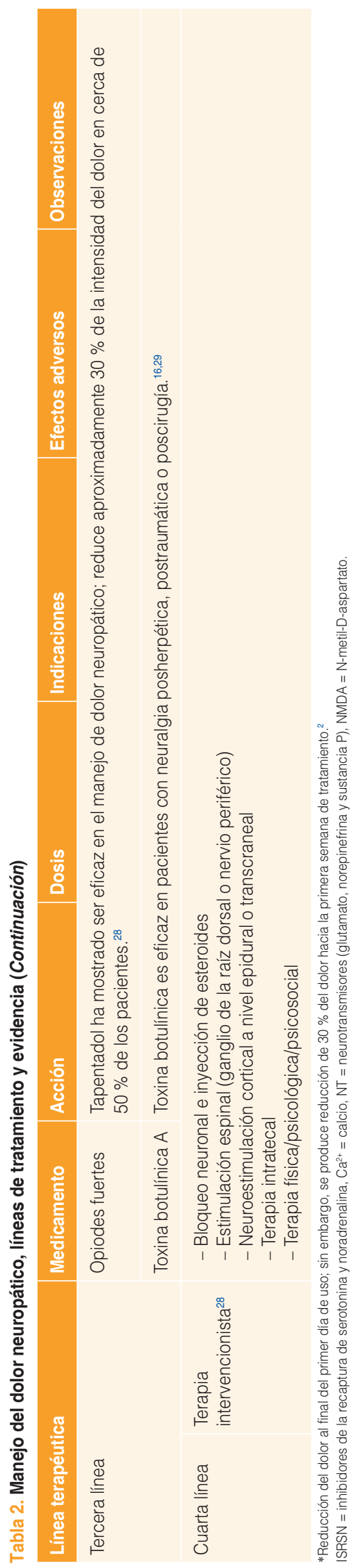

- El riesgo de sobredosis e interacciones medicamentosas es mucho menor que con los fármacos de administración oral. ${ }^{2}$

- Reduce la polifarmacia en los pacientes, sobre todo en los adultos mayores. ${ }^{2,32}$

En el tratamiento del DLN se han usado anestésicos, relajantes musculares, antiinflamatorios, antidepresivos o la combinación de estos fármacos; sin embargo, algunos de ellos no cuentan con el nivel de evidencia que sustente su uso en este tipo de dolor (Figura 2). ${ }^{16,33}$

Algunos agentes tópicos que se utilizan en el tratamiento del DNL son:

- Emulsión de lidocaína a 2.5\% y prilocaína a $2.5 \%$, que constituye el anestésico tópico de mayor uso en el manejo del DLN. ${ }^{2}$

- Parche de lidocaína a 5 \% (tamaño de 10 a $14 \mathrm{~cm}$, con $700 \mathrm{mg}$ de lidocaína), fármaco tópico de primera línea que bloquea los canales de sodio y estabiliza las membranas neuronales (aumento del umbral eléctrico), con lo que se reduce la actividad de las descargas ectópicas y mejora la transducción del dolor. ${ }^{2,8,34}$ La dosis recomendada, dependiendo de la zona anatómica afectada, es de uno a tres parches durante 12 horas, con la que se obtienen resultados entre las dos y cuatro semanas. ${ }^{6,35} \mathrm{Su}$ uso está indicado en el tratamiento de la neuralgia posherpética (con alta eficacia), polineuropatía diabética, síndrome del túnel carpiano, síndrome de dolor miofascial, dolor secundario a osteoartritis y dolor crónico posoperatorio. ${ }^{36,37}$

En varios reportes de casos en pacientes con diagnóstico de neuralgia posherpética se observó 60 a $70 \%$ de reducción del área del dolor después de tres meses de tratamiento con parches de lidocaína a $5 \% .^{16,38}$

Numerosos pacientes han reportado el uso de lidocaína tópica a $5 \%$ por más de ocho años, sin que hayan presentado efectos adversos. ${ }^{2}$ Entre sus efectos adversos, que en forma globlal se presentan en menos de $3 \%$, se encuentran eritema, prurito, edema o irritación cutánea leves en la zona de aplicación. ${ }^{2}$

Otros anestésicos tópicos con bupivacaína o mepivacaína han sido probados en animales; se ha observado reducción de la hiperalgesia en comparación con lidocaína, aunque continúan en protocolos de estudios. $^{16}$

Ketamina es un anestésico parenteral que ofrece analgesia a dosis anestésicas subóptimas: ${ }^{16}$ su acción es a través del bloqueo no competitivo de receptores NMDA (N-metil-D-aspartato), que ocasiona la 


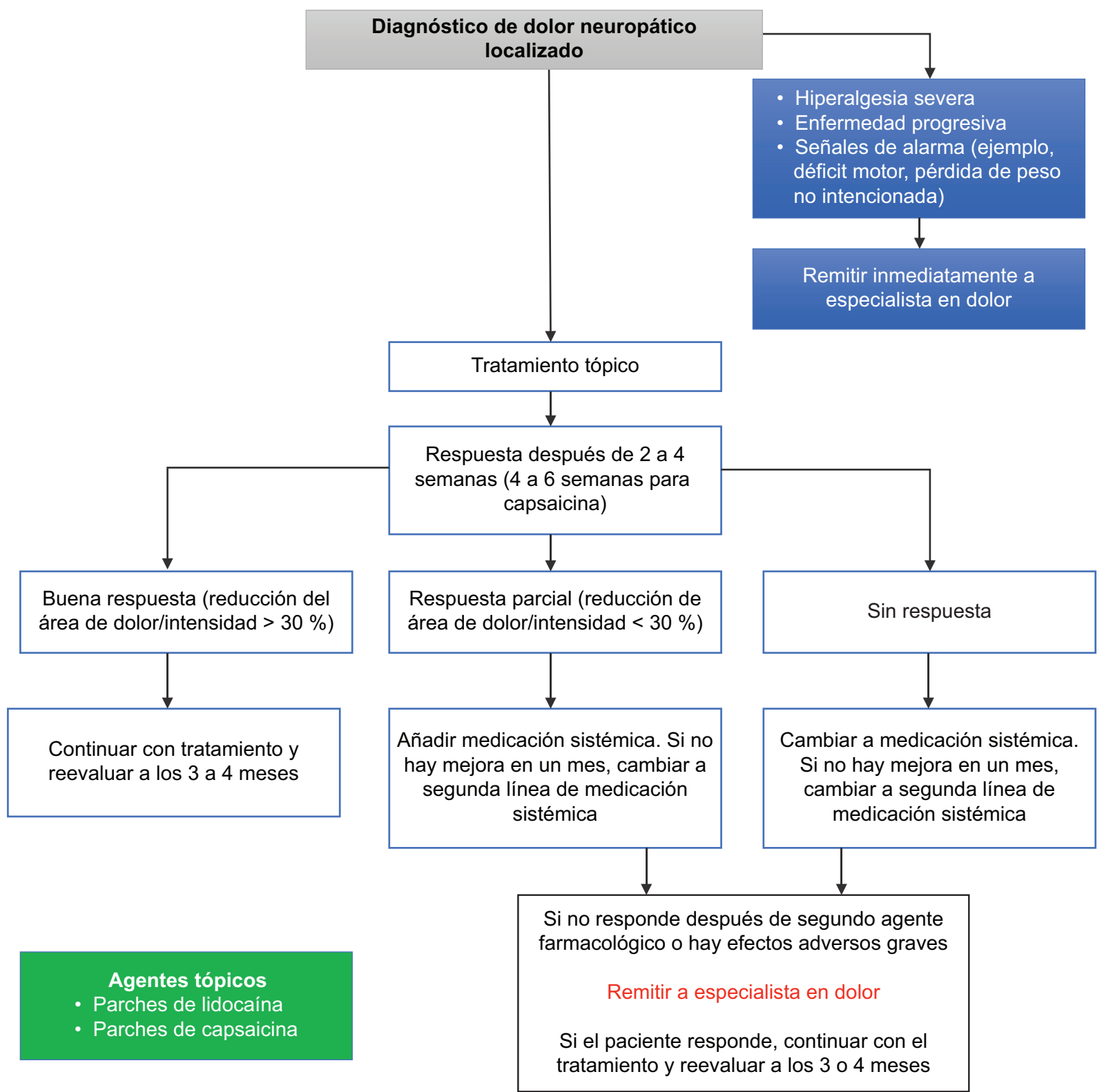

Figura 2. Algoritmo del diagnóstico y tratamiento del dolor neuropático localizado. Basado en Likar $R$ et al.: Treatment of localized neuropathic pain of different etiologies with the $5 \%$ lidocaine medicated plaster - a case series. ${ }^{33}$

modulación del dolor al bloquear la síntesis del glutamato. ${ }^{16}$ La aplicación de ketamina en gel a 0.5-1 \% en pacientes con neuropatía posherpética, polineuropatía diabética y dolor secundario a trauma no ha demostrado eficacia; sin embargo, en pacientes con síndrome doloroso regional complejo se observó reducción de la hiperalgesia y alodinia posterior a la aplicación de ketamina a $10 \%$. 16,38

Baclofeno es un relajante muscular, agonista del receptor $\mathrm{GABA}_{\mathrm{B}}$, que reduce el impulso nervioso a nivel de las vías aferentes mediante la hiperpolarización celular (incremento de iones de potasio y disminución de iones de calcio a nivel intracelular). ${ }^{16}$ En estudios con seres humanos, el baclofeno tópico a $5 \%$ como monoterapia en el manejo del dolor neuropático relacionado con acromegalia y su uso combinado en el tratamiento de dolor neuropático secundario a radiculopatía por una hernia dural lumbar ha demostrado eficacia en la reducción del dolor, con un nivel de evidencia alto..$^{16,38}$

En cuanto a los antiinflamatorios no esteroideos tópicos, el diclofenaco, con una acción a nivel periférico, puso de manifiesto buenos resultados en el tratamiento del dolor neuropático de localización 
orofacial tras su administración tópica a $5 \%$, con un nivel de evidencia moderado; la aplicación es segura sin efectos adversos dermatológicos. ${ }^{16,39} \mathrm{El}$ uso de salicilatos tópicos (aspirina y dietiléter) han resultado excelentes en el manejo de la neuralgia posherpética, con nivel de evidencia moderado. ${ }^{16}$

Respecto a los antidepresivos tricíclicos tópicos, la amitriptilina es un inhibidor de la recaptura de serotonina y noradrenalina mediante el bloqueo de canales dependientes de voltaje de sodio, potasio y calcio, así como de los receptores muscarínicos, colinérgicos, nicotínicos, histaminérgicos, alfa-2 adrenérgicos y receptores NMDA. ${ }^{16,23,38}$ Los resultados han sido ambiguos con amitriptilina en concentraciones de 1 a $5 \%$ en el tratamiento del dolor posherpético, neuropatía diabética o postraumática; con amitriptilina a 5-10\% en el manejo del dolor por polineuropatía axonal idiopática y neuropatía postraumática su obtuvo un nivel de evidencia bajo. ${ }^{16}$ Concentraciones mayores a $10 \%$ podrían llegar a ser efectivas en el manejo del dolor, sin embargo, se han observado efectos adversos similares a los producidos de forma sistémica. ${ }^{13,16}$

Ambroxol, agente mucolítico, posee efectos anestésicos a través del bloqueo de canales de sodio dependientes de voltaje, 40 veces más potente que la lidocaína. Si bien se ha observado reducción de la alodinia en el DLN cuando se aplica tópicamente, los resultados no son concluyentes..$^{16,39}$ En un reporte de casos, la loperamida a $5 \%$ en crema fue efectiva para el manejo de dolor neuropático intratable. ${ }^{39}$

\section{Conclusiones}

Los síndromes de dolor neuropático son observados de forma frecuente en diferentes escenarios clínicos. DNL representa $60 \%$ de las condiciones de dolor neuropático $0^{6} \mathrm{y}, \sin$ duda, afecta la calidad de vida de quienes lo padecen. ${ }^{5}$

Por tal motivo, es importante que el personal médico identifique los diferentes escenarios del dolor neuropático, incluidos los síndromes de DNL, para el cual se pueden ofrecer otros esquemas farmacológicos, como los tratamientos tópicos, principalmente en personas de edad avanzada que ya reciben múltiples fármacos. ${ }^{2,3,5,9}$

\section{Conflicto de intereses}

Los autores declaran no tener conflicto de interés alguno.

\section{Financiamiento}

La presente investigación no recibió ninguna beca específica de agencias de los sectores públicos, comercial o sin ánimo de lucro.

\section{Responsabilidades éticas}

Protección de personas y animales. Los autores declaran que para esta investigación no se realizaron experimentos en seres humanos ni en animales.

Confidencialidad de los datos. Los autores declaran que en este artículo no aparecen datos de pacientes.

Derecho a la privacidad y consentimiento informado. Los autores declaran que en este artículo no aparecen datos de pacientes.

\section{Bibliografía}

1. Scholz J, Finnerup NB, Attal N, Aziz Q, Baron R, Bennett MI, et al. The IASP classification of chronic pain for ICD-11: chronic neuropathic pain. Pain. 2019;160:53-59.

2. Allegri M, Baron R, Hans G, Correa-Illanes G, Mayoral-Rojals V, Mick G, et al. A pharmacological treatment algorithm for localized neuropathic pain. Curr Med Res Opin. 2016:32:377-384

3. Mick G, Baron R, Finnerup NB, Hans G, Kern KU, Brett B, et al. What is localized neuropathic pain? A first proposal to characterize and define a widely used term. Pain Manag. 2012;2:71-77.

4. Finnerup NB, Haroutounian S, Kamerman P, Baron R, Bennett DLH, Bouhassira D, et al. Neuropathic pain: an updated grading system for research and clinical practice. Pain. 2016;157:1599-1606.

5. Colloca L, Ludman T, Bouhassira D, Baron R, Dickenson AH, Freeman R, et al. Neuropathic pain. Nat Rev Dis Prim. 2017;3:1-20.

6. Pickering G, Martin E, Tiberghien F, Delorme C, Mick G. Localized neuropathic pain: an expert consensus on local treatments. Drug Des Devel Ther. 2017;11:2709-2718.

7. Mayoral V, Pérez-Hernández C, Muro I, Leal A, Villoria J, Esquivias A. Diagnostic accuracy of an identification tool for localized neuropathic pain based on the IASP criteria. Curr Med Res Opin. 2018;34:1465-1473.

8. Kraychete DC, Sakata RK. Painful peripheral neuropathies. Rev Bras Anestesiol. 2011;61:649-658.

9. Eastman RC. Neuropathy in diabetes. En: Diabetes research and clinical practice. 1995;30:75.

10. Mick G, Baron R, Correa-Illanes G, Hans G, Mayoral V, Frías X, et al. Is an easy and reliable diagnosis of localized neuropathic pain (LNP) possible in general practice? Development of a screening tool based on IASP criteria. Curr Med Res Opin. 2014;30:1357-1366.

11. Golan-Vered Y, Pud D. Chemotherapy-induced neuropathic pain and its relation to cluster symptoms in breast cancer patients treated with paclitaxel. Pain Pract. 2013;13:46-52.

12. Casale R, Mattia C. Building a diagnostic algorithm on localized neuropathic pain (LNP) and targeted topical treatment: Focus on $5 \%$ lidocaine-medicated plaster. Ther Clin Risk Manag. 2014;10:259-268.

13. Hatch MN, Cushing TR, Carlson GD, Chang EY. Neuropathic pain and SCl: Identification and treatment strategies in the $21^{\text {st }}$ century. $J$ Neurol Sci. 2018;384:75-83.

14. Treede RD, Jensen TS, Campbell JN, Cruccu G, Dostrovsky JO, Griffin JW, et al. Neuropathic pain: redefinition and a grading system for clinical and research purposes. Neurology. 2008;70:1630-1635.

15. Guy SD, Mehta S, Casalino A, Côté I, Kras-Dupuis A, Moulin DE, et al. The CanPain $\mathrm{SCl}$ clinical practice guidelines for rehabilitation management of neuropathic pain after spinal cord: recommendations for treatment. Spinal Cord. 2016;54:S14-S23.

16. Dworkin RH, O'Connor AB, Backonja M et al. Pharmacologic management of neuropathic pain: evidence-based recommendations. Pain. 2007:132:237-251.

17. Moulin DE, Boul, er A, et al. Pharmacological management of chronic neuropathic pain - Revised consensus statement from the Canadian pain society. Pain Res Manag. 2014:19(6):e87.

18. Sommer C, Cruccu G. Topical Treatment of Peripheral Neuropathic Pain: Applying the Evidence. J Pain Symptom Manage. 2017;53(3):614-629. DOI:10.1016/j.jpainsymman.2016.09.015 
19. Bistre S, Acevedo JC, Amaya A, et al. Guidelines for the diagnosis and management of neuropathic pain: Consensus of a group of latin american experts. J Pain Palliat Care Pharmacother. 2009;23(3):261-281. DOI:10.1080/15360280903098572

20. Cruccu G, Truini A. A review of Neuropathic Pain: From Guidelines to Clinical Practice. Pain Ther. 2017;6(S1):35-42. DOI:10.1007/s40122-017-0087-0

21. Haanpää M, Attal N, Backonja M, et al. NeuPSIG guidelines on neuropathic pain assessment. Pain. 2011;152(1):14-27. DOI:10.1016/i.pain.2010.07.031

22. Dworkin RH, O'Connor AB, Audette J, et al. Recommendations for the pharmacological management of neuropathic pain: An overview and literature update. Mayo Clin Proc. 2010;85(3):S3-S14. DOI:10.4065/mcp.2009.0649

23. Kremer M, Salvat E, Muller A, Yalcin I, Barrot M. Antidepressants and gabapentinoids in neuropathic pain: Mechanistic insights. Neuroscience. 2016;338:183-206. DOI:10.1016/j.neuroscience.2016.06.057

24. Attal N, Cruccu G, Haanpää M, et al. EFNS guidelines on pharmacological treatment of neuropathic pain. Eur J Neurol. 2006;13(11):11531169. DOI:10.1111/j.1468-1331.2006.01511.x

25. Attal N, Cruccu G, Baron R, et al. EFNS guidelines on the pharmacological treatment of neuropathic pain: 2010 revision. Eur J Neurol. 2010;17(9):1113-1123. DOI:10.1111/j.1468-1331.2010.02999.x

26. Treede RD, Wagner T, Kern KU, Arendt G, Birklein F, Cegla T, et al. Mechanism- and experience-based strategies to optimize treatment response to the capsaicin $8 \%$ cutaneous patch in patients with localized neuropathic pain. Curr Med Res Opin. 2013;29:527-538.

27. Pinto JT, Pereira FC, Loureiro MC, Gama R, Fernandes HL. Efficacy analysis of capsaicin $8 \%$ patch in neuropathic peripheral pain treatment. Pharmacology. 2018;101:290-297.

28. Casale R, Symeonidou Z, Bartolo M. Topical treatments for localized neuropathic pain. Curr Pain Headache Rep. 2017;21:15.

29. Park JH, Park HJ. Botulinum toxin for the treatment of neuropathic pain. Toxins (Basel). 2017;9:260.
30. Kopsky DJ. Extending the therapeutic scope for the treatment of neuropathic pain with topical analgesics. J Pain Reli. 2016:5:3-5.

31. Peppin JF, Albrecht PJ, Argoff C, Gustorff B, Pappagallo M, Rice FL, et al. Skin matters: a review of topical treatments for chronic pain. part one: skin physiology and delivery systems. Pain Ther. 2015;4:17-32.

32. Pickering G, Lucchini $C$. Topical treatment of localized neuropathic pain in the elderly. Drugs Aging. 2020;37:83-89.

33. Lara-Solares A, Mayoral-Rojals V Guillén-Núñez MR, Villafaña-Tello JJS, Cabtú-Brito C, Genis-Rondero MA, et al. Consenso multidisciplinario de diagnóstico y tratamiento del dolor neuropático periférico y localizado en México. Gac Med Mex. 2019;155:423-427.

34. Likar R, Demschar S, Kager I, Neuwersch S, Pipam W, Sittl R. Treatment of localized neuropathic pain of different etiologies with the $5 \%$ lidocaine medicated plaster - a case series. Int J Gen Med. 2014;8:9-14.

35. Buksnys T, Armstrong N, Worthy G, Sabatschus I, Boesl I, Swift SL, et al. Systematic review and network meta-analysis of the efficacy and safety of lidocaine $700 \mathrm{mg}$ medicated plaster vs. pregabalin. Curr Med Res Opin. 2020;36:101-115.

36. Schreiber AK, Nones CFM, Reis RC, Chichorro JG, Cunha JM. Diabetic neuropathic pain: physiopathology and treatment. World J Diabetes. 2015;6:432.

37. Palladini M, BoesI I, Koenig S, Buchheister B, Attal N. Lidocaine medicated plaster, an additional potential treatment option for localized post-surgical neuropathic pain: efficacy and safety results of a randomized, placebo-controlled trial. Curr Med Res Opin. 2019;35:757-766.

38. Knezevic NN, Tverdohleb T, Nikibin F, Knezevic I, Candido KD. Management of chronic neuropathic pain with single and compounded topical analgesics. Pain Manag. 2017:7:537-558.

39. Coderre TJ. Topical drug therapeutics for neuropathic pain. Expert Opin Pharmacother. 2018;19:1211-1220 